\title{
Differential accumulation of advanced glycation end products in the course of diabetic retinopathy
}

\author{
H.-P. Hammes ${ }^{1}$, A. Alt ${ }^{1}$, T. Niwa ${ }^{2}$, J.T. Clausen ${ }^{3}$, R. G. Bretzel ${ }^{1}$, M. Brownlee ${ }^{4}$, E. D. Schleicher ${ }^{5}$ \\ ${ }^{1}$ Justus-Liebig-Universität Giessen, Giessen, Germany \\ ${ }^{2}$ Nagoya University, Daiko Medical Centre, Nagoya, Japan \\ ${ }^{3}$ Bio Sciences, Novo Nordisk A/L, Bagsvaerd, Denmark \\ ${ }^{4}$ Diabetes Research Center, Albert Einstein College of Medicine, Bronx, New York, USA \\ ${ }^{5}$ Department of Internal Medicine, Division of Endocrinology, Metabolism and Pathobiochemistry, Tübingen, Germany
}

\section{Abstract}

Aims/hypothesis. Glycated proteins, formed by reaction of glucose and protein, react further yielding numerous, mostly undefined advanced glycation end products (AGE). The recently characterized imidazolone-type AGE (AG-1) is non-oxidatively formed involving 3-deoxyglucosone whereas some AGEs, particularly $\mathrm{N}^{\varepsilon}$-(carboxymethyl)lysine (CML), are formed only in the presence of oxygen. Methods. To study the possible contribution of oxidative and non-oxidative AGE formation in the development of diabetic retinopathy antibodies directed against CML-type and imidazolone-type AGEs were characterized by dot blot analysis and used to localize these well-characterized epitops in the retinas from diabetic rats (early course) and from human Type I (insulin-dependent) diabetes mellitus with lasertreated proliferative diabetic retinopathy (late course).

Results. In non-diabetic rats CML was moderately positive in neuroglial and vascular structures of nondiabetic rat retinas and increased strongly in diabetic retinas. Anti-imidiazolone antibody staining was strongly positive only in diabetic capillaries. Advanced human diabetic retinopathy showed strong CML-immunolabelling of the entire retina whereas control samples showed moderate staining of neuroglial structures only with the polyclonal CML-antibody. Anti-imidiazolone antibody staining was faint in the inner retina of control sections but were strong throughout the entire diabetic retina. Immunolabelling for the AGE-receptor was congruent with a marker of Müller cells.

Conclusion/interpretation. Our data indicate that the oxidatively formed CML is present in non-diabetic retinas as a regular constituent but increases in diabetes both in neuroglial and vascular components. Imidazolone-type AGE are restricted to microvessels and spread during later stages over the entire retina, co-localizing with the expression of AGE-receptor. [Diabetologia (1999) 42: 728-736]

Keywords Advanced glycation end products, carboxymethyllysine, retinopathy, extracellular matrix, oxidative stress.
Received: 22 October 1998 and in revised form: 11 January 1999

Corresponding author: Dr. H. P. Hammes, 3rd Medical Department, Justus-Liebig-Universität, Rodthohl 6, D-35392 Giessen, Germany

Abbreviations: AGE, Advanced glycation end products; CML, $\mathrm{N}^{\mathrm{e}}$-(carboxymethyl)lysine; CEL, $\quad \mathrm{N}^{\varepsilon}$-(carboxyethyl)lysine; AG-1, anti-imidazolone antibody; RAGE, receptor for advanced glycation end products; GFAP, glial fibrillary acid protein.
Mean increase of blood glucose concentrations in patients with diabetes mellitus has been implicated in the development of degenerative microvascular changes $[1,2]$. One mechanism linking chronic hyperglycaemia with tissue damage such as that in diabetic retinopathy is the formation and accumulation of advanced glycation end products (AGE) [3]. Studies done in vitro and in vivo have indicated that AGE induce irreversible crosslinks in long-living extracellular matrix [4-8] and, upon binding to specific cellular proteins, change the local concentrations of cytokines, growth factors and other bioactive molecules 
[9-11]. Advanced glycation end products accumulate with age and, at an accelerated rate, in diabetes [12-14]. Fluorescent AGEs in the retinas of diabetic animals correlate with the duration and degree of hyperglycaemia [15]. Fluorescent AGEs were first described by us to accumulate in precapillary arteriolar branching sites in retinas of diabetic rats [15].

Advanced glycation end products are a heterogenous group and it is not known how many there are of them [16]. Up to now only a few products occurring in vivo have been fully characterized and are therefore structurally well-defined [17]. After reaction of glucose with the amino groups of proteins and subsequent Amadori rearrangement a variety of chemically different structures are formed [16]. Advanced glycation end products are formed non-oxidatively through highly reactive carbonyl compounds such as 3-deoxyglucosone which reacts with free amino groups. It has been shown that when 3-deoxyglucosone reacts with lysine, pyrraline is formed [18] whereas with arginine it yields a hydroimidazolone $[19,20]$. Other candidate compounds of AGE include a group which form through oxidative cleavage of the carbohydrate moiety yielding mainly $\mathrm{N}^{\varepsilon}$-(carboxymethyl)lysine (CML) and erythronic acid [21-24]. As this reaction occurs only in the presence of oxygen, the resulting product CML was named a glycoxidation product. The fluorescent pentosidine represents another well-characterized oxidatively formed product representing a minor proportion of the AGEs [7, 8]. Furthermore, AGEs may be formed from auto-oxidation products of glucose [25].

The use of antibodies against AGE structures led to the discovery that only a minor proportion of AGE are detectable by autoflourescence and that they form intracellularly much faster and to a greater extent than extracellularly because several glucose fragmentation products which occur during the metabolism of glucose in the cell are more reactive than glucose itself [26]. It was also found that the non-flourescent CML is the major epitope against which AGE-antibodies are directed [23, 27, 28]. Some AGE-antibodies used so far have not been characterized at all. The currently available AGE-antisera therefore contain variable amounts of antibodies against undefined AGE-epitopes. To circumvent this problem we applied antibodies directed against the well-characterized epitops CML and AG-1 as representative markers for the oxidative and non-oxidative pathway, respectively.

Part of the pathogenic reaction excerted by AGEs is mediated by interaction with cellular receptors. One of them has been termed RAGE and mediates both adhesive as well as procoagulant properties of AGEs [29, 30]. The interaction of AGE with RAGE is of crucial importance for the understanding of the pathobiochemical role of AGE in diabetic angiopathy.
Our aim was to investigate the possible contribution of AGE formation in the development of diabetic retinopathy. We used rat retinal tissues as a model of very early retinopathy with similarities to the alterations in human retinas, such as pericyte drop-out, increased endothelial cell numbers and the formation of acellular capillaries. In addition, autoptic material of human proliferative diabetic retinopathy representing the other end of the natural course of microvascular damage was studied. As several cellular binding sites for AGE were discovered on a variety of cells such as endothelial cells and smooth muscle cells, we investigated the co-localization of the AGE with RAGE.

\section{Materials and methods}

Proteins and antibodies. We prepared CML-BSA, AGE-modified BSA and CML-keyhole limpit haemocyanin (KLH) as described previously $[14,28]$. We also prepared $\mathrm{N}^{\varepsilon}$-(carboxyethyl)lysine (CEL) as described previously [31] and characterized it by mass spectrometry. For the detection of tissue-bound imidazolone-type AGE and CML-type glycoxidation products, we used only antibodies whose epitopes had been characterized previously. The monoclonal anti-CML antibody (6D12) and the monoclonal anti-imidazolone antibody (AG-1) were prepared and characterized as described in [28] and [19,20], respectively. The polyclonal anti-CML antibody was generated in rabbits and extensively characterized [14]. The RAGE antibody was generated in rabbits using the extracellular N-terminal part of the RAGE (amino acid sequence GAAGTAVGAWVLVLS) [32], emulsified in Freund's complete adjuvans, as antigen. The antibody was characterized by western blotting using membranes from Hek 293 cells transfected with RAGE. A single band was observed in retinal extracts from normal and diabetic rats at a molecular weight level of $\sim 39 \mathrm{kDa}$, while bands with a molecular weight between 45 and $52 \mathrm{kDa}$ were observed in samples from transfected cell membranes (due to different posttranslational glycosylation of RAGE in Hek cells) whereas no bands were detected in the non-transfected cells (not shown). The polyclonal antibody against glial fibrillary acid protein (GFAP) was obtained from Dako (Hamburg, Germany).

Dot blot analysis of the antibody affinity. We loaded $0.5 \mu \mathrm{l}$ of increasing concentrations ranging from 1 to $1000 \mathrm{ng} / \mathrm{ml}$ of each antigen on a nitrocellulose membrane (Hybond-N +, Amersham, Braunschweig, Germany) and dried them at room temperature for $10 \mathrm{~min}$. Membranes were washed, blocked with $5 \%$ non-fat dry milk for $30 \mathrm{~min}$, washed again and incubated using the following antibody concentrations: monoclonal CML antibody (6D12) $0.1 \mu \mathrm{g} / \mathrm{ml}$, monoclonal imidazolone-type AGE antibody (AG-1) $0.1 \mu \mathrm{g} / \mathrm{ml}$ and the polyclonal CML antibody diluted 1:10000. With the latter dilution we aimed to obtain a similar antibody concentration as the monoclonal antibodies based on the assumption that less than $0.01 \%$ of total $\mathrm{IgG}$ recognizes the specific antigen. After extensive washing in PBS-T, membranes were exposed to peroxidase-labelled antimouse IgG or peroxidase-labelled anti-rabbit IgG antbodies (Dako, Hamburg, Germany), respectively, for $1 \mathrm{~h}$. Membranes were washed again and exposed to the enhanced chemoluminiscence detection system (Amersham Buchler, Braunschweig, Germany) according to the manufacturer's instructions. Membranes were subsequently exposed to x-ray film (Sterling, New- 
ark, Del., USA). Developed films were scanned into an image analysis system (CUE-2, Olympus Opt. Europe, Hamburg, Germany) and optical density and areas of the dots were quantified. The results were obtained from three independent experiments and three independent determinations of each individual AGE value. Results were expressed as arbitrary intensity units of optical densities (AIU).

Human diabetic and control retinas. We studied the immunolocalization of CML and AGE in an eye with advanced diabetic disease from a patient in whom diabetes duration had been strictly defined and who had no concomittant eye disease. Therefore we restricted our study to one eye which we obtained after death from a 43-year-old patient with Type I (insulin-dependent) diabetes mellitus (duration 39 years) and proliferative diabetic retinopathy who had been treated with panretinal photocoagulation 10 years ago. For controls, five eyes were obtained from autopsies (three men and two women, age 42-79 years) without diabetes or any known ocular disease. Eyes were fixed in $4 \%$ PBS-buffered formalin and $6 \mu \mathrm{m}$ vertical sections were made of the paraffin-embedded samples.

Immunohistochemistry. Sections were incubated with the 6D12 antibody $(2 \mu \mathrm{g} / \mathrm{ml})$ and the AG-1 antibody $(10 \mu \mathrm{g} / \mathrm{ml})$ for $1 \mathrm{~h}$ at room temperature. After incubation with the bridging antibody the secondary anti-alkaline phosphatase labelled antibody was added for 20 min followed by substrate incubation (New Fuchsin, Dako) and counterstaining with hemalaun. The polyclonal CML-antibody was used according the previously described method [14]. Briefly, sections were incubated in $3 \% \mathrm{H}_{2} \mathrm{O}_{2}$ in TRIS-buffered saline (TBS) to block endogenous peroxidase. After repeated rinses in TBS, sections were incubated with normal pig serum at a 1:5 dilution in TBS to reduce unspecific binding. After rinsing in TBS, sections were incubated with the polyclonal antibody against the RAGE (dilution 1:150) for $1 \mathrm{~h}$ at room temperature. After exposure to the appropriate bridging and secondary antibody, tissues were made visible using 3'3'diaminobenzidine hydrochloride as chromogen. Sections were counterstained with hemalaun. Glial fibrillary acid protein (GFAP) was localized using a polyclonal antibody (Dako, $4.1 \mathrm{mg} / \mathrm{ml}$, dilution 1:100) as described previously [33].

Diabetic and non-diabetic rats. The study conformed with the Association for Research in Vision and Ophthalmology resolution of use of animals in research. Six week-old male Wistar rats (Charles River, Sulzfeld, Germany) were rendered diabetic by i. v. injection of streptozotocin $(65 \mathrm{mg} / \mathrm{kg}$ body weight; Boehringer Mannheim, Mannheim, Germany). Animals with blood glucose values lower than $15 \mathrm{mmol} / \mathrm{l}$ after 1 week were excluded from the study. After 6 months of diabetes, eyes were enucleated from the diabetic group together with eyes from non-diabetic animals and immediately stored in liquid nitrogen.

Vertical and horizontal cryostat sections $(6 \mu \mathrm{m})$ were prepared as described previously and stained with the primary antibodies described above, except that an immunofluorescence protocol was used for GFAP staining [3].

\section{Results}

Dot blot analysis. On immuno blot analysis the AGEantibodies used in this study showed different reactivity against highly modified AGE-BSA and highly CML-modified proteins (Fig.1). The polyclonal CML antibody recognized all three antigens with similar reactivity, indicating the presence of CML in the

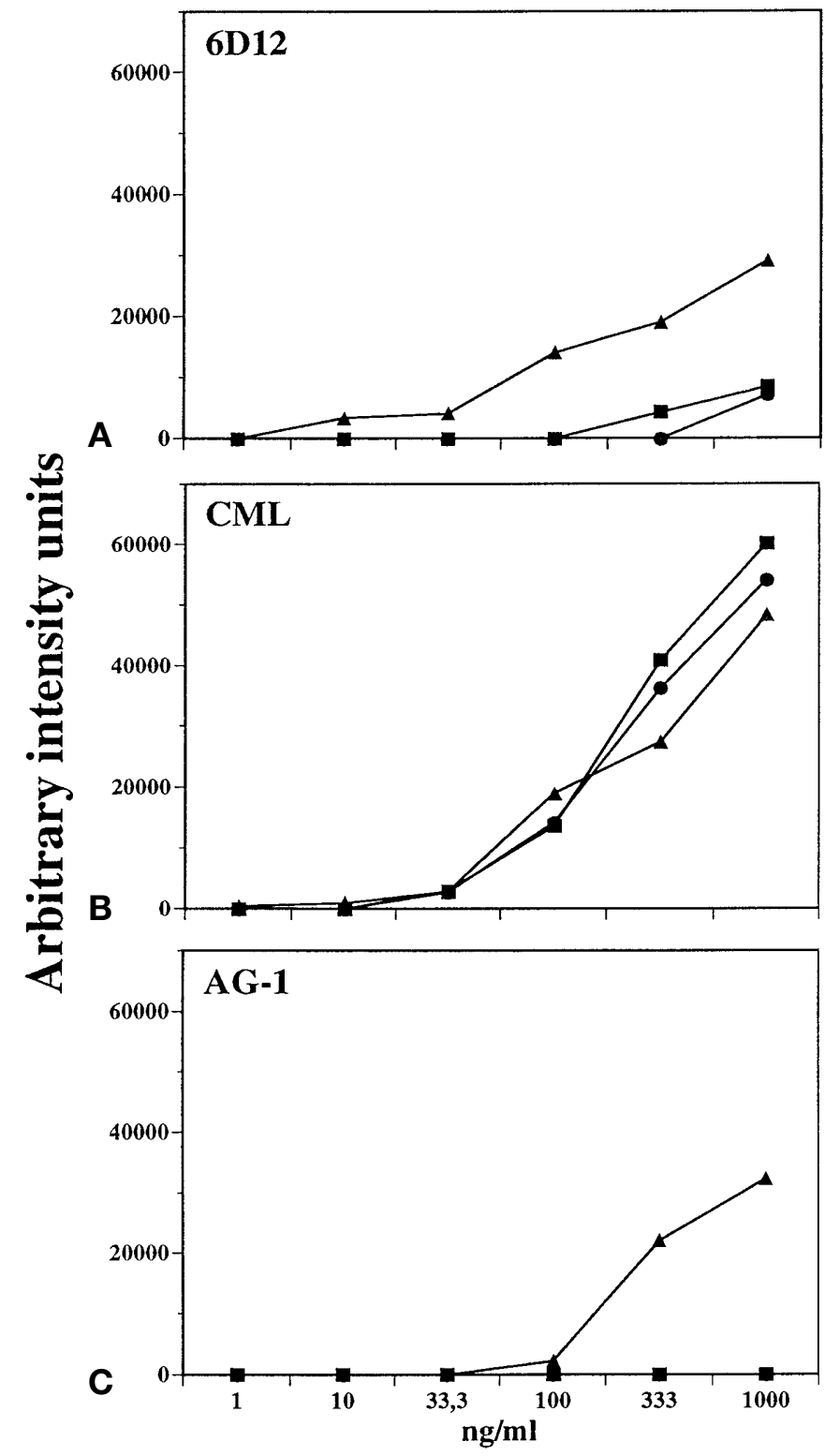

Fig. 1. A-C Immunoreactivity of the CML-antibodies and imidazolone-antibodies to the highly-modified AGE-proteins CML-KHL, CML-BSA and AGE-BSA. A monoclonal CMLantibody (6D12); B polyclonal CML antibody; C AG-1 antibody. Membranes were loaded with increasing amounts of antigen and antibodies-binding was determined as described in the method section. On the y-axis the arbitrary intensity units of optical densities intensities (AIU) are indicated. Results are expressed as the means of three independent experiments. The individual values generally scattered less than $7.4 \%$ from the mean of each measurement ( - CML-KLH, - CMl$\mathrm{BSA}, \boldsymbol{\sim}$ AGE-BSA)

AGE-BSA preparation. In contrast, the monoclonal antibody 6D12 started to react against much higher concentrations ( $\sim$ tenfold) of AGE-BSA compared with the polyclonal CML antibody and was also less reactive against both CML-preparations. Note that both CML antibodies used did not recognize CEL (1-1000 $\mathrm{ng} / \mathrm{ml}$ ) in our assay (data not shown) since 
$\mathbf{N}$

D

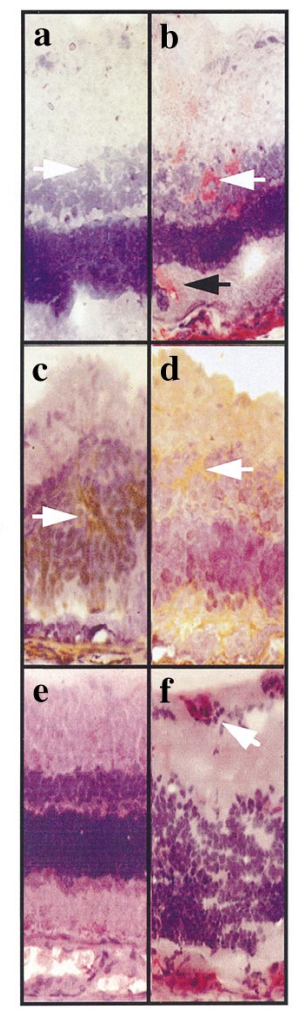

$\mathbf{N}$

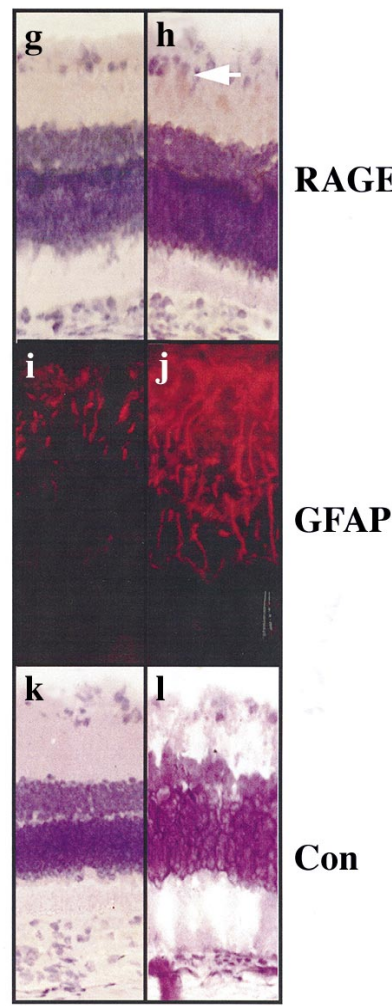

Fig. 2.a-l Immunolocalization of AGE and RAGE in vertical sections of rats with early experimental diabetic retinopathy. CML immunlocalization using a monoclonal (6D12) and a polyclonal (CML) antibody, compared with the imidazolonetype AGE (AG-1) and RAGE in normal (N) and diabetic (D) rats. $\mathbf{a}$ and $\mathbf{b}$ : white arrows indicate immunolabelling in the inner nuclear layer, black arrow in $\mathbf{b}$ indicates CML labelling in the photoreceptor layer. c and $\mathbf{d}$ : white arrow indicates positive labelling in the inner nuclear layer. e: no immunoreactivity for the 3-deoxyglucosone derived in non-diabetic rat retina. f: positive labelling in the vessels of the ganglion cell layer (white arrow). g: mild labelling for RAGE in the inner nuclear layer. h: Moderate RAGE immunoreactivity in the inner plexiform layer (white arrow). i: GFAP labels astrocytes in the normal rat retina. j: GFAP labels astrocytes and Müller cells in the diabetic rat retina. $\mathbf{k}$, I negative controls. Sections were counterstained with hematoxylin. Original magnification $25 \times$

this structurally closely related modification is formed non-oxidatively [34]. The imidazolone antibody (AG1) did not react with the two CML preparations used but recognized AGE-BSA at concentrations as low as $100 \mathrm{ng} / \mathrm{ml}$. No immunoreaction was detected with nonimmune mouse or rabbit IgG. By comparing reactivities of the various antibodies used therefore it became evident that AG-1 antibody was solely reactive against non-CML epitopes in AGE-BSA samples. Thus, the 6D12 antibody is a medium affinity CMLprotein-recognizing antibody and the polyclonal CML antibody is a high affinity antibody.

Immunohistochemistry in experimental diabetic retinopathy (early course). Both, vertical and horizontal

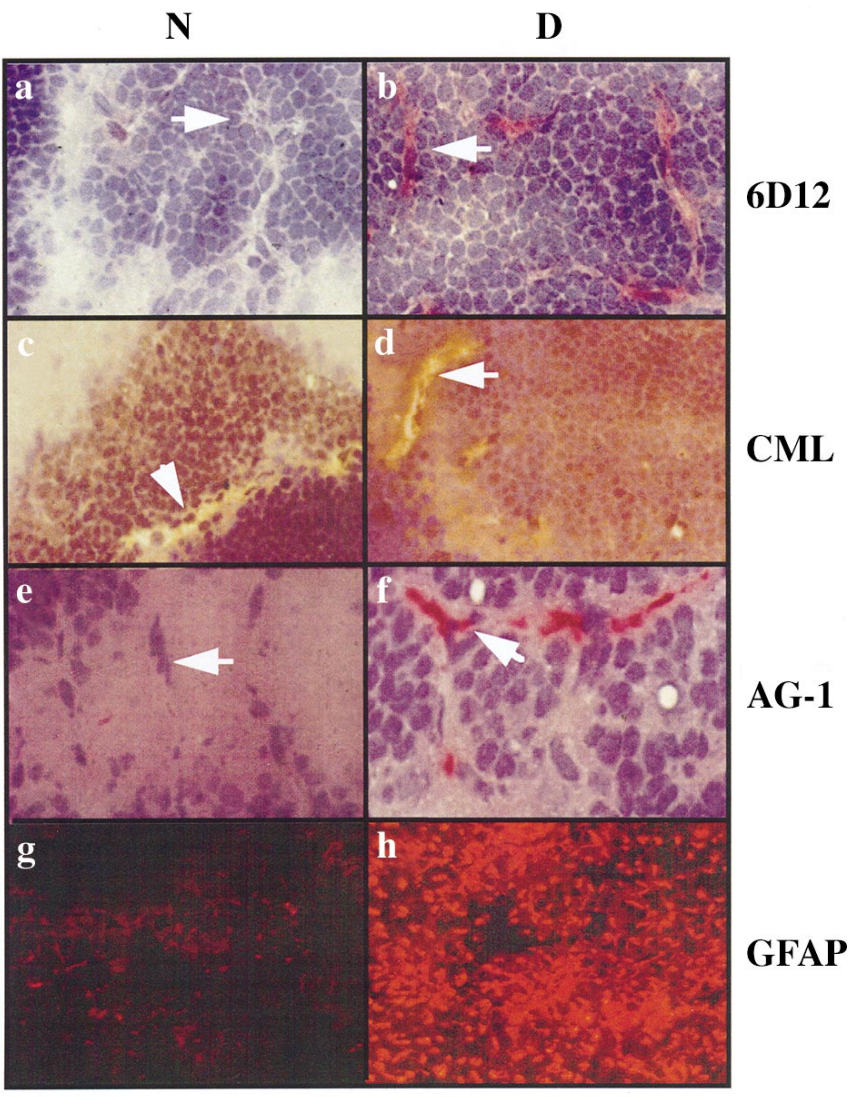

Fig. 3.a-h Immunolocalization of AGE and CML in comparison with GFAP in horizontal sections of normal $(\mathrm{N})$ and diabetic (D) rats with early experimental diabetic retinopathy. a: mild immunoreactivity of CML in capillaries of the inner nuclear layer (arrow). b: CML immunoreactivity in capillaries of the inner nuclear layer (arrow) and in pericapillary areas of the inner retina. c: CML immunoreactivity (polyclonal antibody) in the vascular (arrow) and the non-vascular structures of the inner nuclear layer. d: Immunoreactivity in diabetic rats. Strong signal in the capillaries of the inner retina (arrow) and the perivascular areas of the inner nuclear layer. e: No AG-1 immunoreactivity in the normal retina. f: AG-1 immunoreactivity limited to capillaries of the inner nuclear layer. $\mathbf{g}$, $\mathbf{h}$ : GFAP in normal (g) and diabetic (h) rat retina. Original magnification $25 \times$

crystat sections were used to identify the spatial distribution of the antibodies and to investigate their relative co-localization with RAGE and a marker of Müller cell transdifferentiation in diabetes, GFAP. In non-diabetic rats, 6D12-immunoreactivity was weakly positive in the inner plexiform and inner nuclear layer and, in particular, positive in capillaries of the inner nuclear layer (Figs. $2 \mathrm{a}, 3 \mathrm{a}$ ). In diabetic rat retinas, the immunoreactivity was predominantly increased in the inner nuclear layer (Fig. 2b) and in capillaries (Fig. 3 b). Of note, CML immunoreactivity was also increased in the photoreceptor layer.

The polyclonal anti-CML antibody specifically stained the non-diabetic retina from the inner limiting membrane to the outer border of the inner nucle- 
Table 1. Immunohistochemical detection of AGE products in retinas of normal and diabetic rats

\begin{tabular}{|c|c|c|c|c|c|c|}
\hline & \multicolumn{6}{|c|}{ antibody } \\
\hline & \multicolumn{2}{|c|}{ 6D12 } & \multicolumn{2}{|c|}{ CML } & \multicolumn{2}{|c|}{ AG-1 } \\
\hline & $\mathrm{N}$ & D & $\mathrm{N}$ & D & $\mathrm{N}$ & D \\
\hline $\mathrm{GC} / \mathrm{NFL}$ & 0 & + & ++ & ++++ & 0 & 0 \\
\hline IPL & + & +++ & ++ & ++++ & 0 & 0 \\
\hline INL & + & +++ & ++ & ++++ & 0 & 0 \\
\hline OPL & 0 & ++ & + & ++ & 0 & 0 \\
\hline ONL & 0 & + & + & ++ & 0 & 0 \\
\hline PR & 0 & +-+++ & ++ & ++++ & 0 & 0 \\
\hline Vessels $^{\mathrm{a}}$ & + & ++++ & ++ & ++++ & 0 & ++++ \\
\hline
\end{tabular}

${ }^{a}$ as determined from the horizontal sections

$\mathrm{N}=$ normal; $\mathrm{D}=$ diabetic. 0 None; + weakly positive; ++ moderately; +++ strongly positive; ++++ intensely stained; GCl NFL, ganglian cell/nerve fibre layer; IPL, inner plexiform layer; INL, inner nuclear layer; OPL, outer plexiform layer; ONI, outer nuclear layer; PR, photoreceptor

Table 2. Immunohistochemical detection of AGE-products in human retinas from normal and diabetic ${ }^{\mathrm{a}}$ retinas

\begin{tabular}{|c|c|c|c|c|c|c|}
\hline & \multicolumn{6}{|c|}{ antibody } \\
\hline & \multicolumn{2}{|c|}{$6 \mathrm{D} 12$} & \multicolumn{2}{|c|}{ CML } & \multicolumn{2}{|c|}{ AG-1 } \\
\hline & $\mathrm{N}$ & $\mathrm{D}$ & $\mathrm{N}$ & $\mathrm{D}$ & $\mathrm{N}$ & $\mathrm{D}$ \\
\hline GC/NFL & 0 & +++ & ++ & +++ & 0 & ++ \\
\hline IPL & 0 & ++++ & + & ++++ & 0 & +++ \\
\hline INL & 0 & ++ & ++ & ++ & 0 & + \\
\hline OPL & 0 & +++ & 0 & ++++ & 0 & 0 \\
\hline ONL & 0 & +++ & 0 & ++ & 0 & 0 \\
\hline PR & 0 & +++ & + & +++ & 0 & 0 \\
\hline
\end{tabular}

The abbreviations are the same as in Table 1

a obtained from a Type I diabetic patient with laser treated proliferative diabetic retinopathy

ar layer and the photoreceptor layer (Fig. 2c). As observed in the horizontal sections (Fig. $3 \mathrm{c}$ ) capillary and pericapillary structures were moderately labelled. A major increase of immunoreactivity was observed in retinas from diabetic rats, predominantly in the inner retina and in the photoreceptor layer (Fig. 2d). It was also evident from the horizontal sections, that diabetic capillaries were strongly positive.

A major difference of the staining pattern was observed with the AG-1 antibody. This antibody did not show any positive reaction in the normal retina (Fig. 2e), particularly no neuroglial staining. The only structures that were labelled were vessels (Figs. $2 \mathrm{f}, 3 \mathrm{f}$ ). Table 1 summarizes results of AGE immunostaining in non-diabetic and diabetic rat retinas.

The antibody against the extracellular part of the RAGE did not recognize any structure in the normal rat retina. No difference was observed compared with negative control sections (Fig. $2 \mathrm{~g}, \mathrm{k}$ ). A moderately positive staining pattern of the inner retina (from the inner limiting membrane to outer border of the inner nuclear layer) was only present in diabetic sections (Fig. 2h). Of note, CML-immunoreactivity did not co-localize with RAGE in the inner retina (i.e. from the inner limiting membrane to the outer border of the inner nuclear layer) and there was no precise colocalization of the CML-antibodies with AG-1. To identify neuroglial structures, GFAP was used. In non-diabetic retina GFAP antibody labelled astrocytes (Figs. 2i, $3 \mathrm{~g}$ ) and in diabetic retinas astrocytes and Müller cells were stained (Figs. $2 \mathrm{j}, 3 \mathrm{~h}$ ).

Immunohistochemistry in advanced human diabetic retinopathy (advanced course). Results of AGE immunostaining in the retina from the Type I diabetic patient with proliferative diabetic retinopathy which had been laser treated are summarized in Table 2.

Non-diabetic retinal tissue did not stain with the 6D12 antibody (Fig. 4a). A strong positive reaction was, however, observed throughout the whole diabetic retina with predominance of the inner plexiform layer (Fig. 4b), comprising positively labelled structures, the inner limiting membrane, ganglion cell layer, inner and outer nuclear layer and photoreceptor layer. Of importance, thickened walls of vessels in the inner retina were also labelled.

As in the non-diabetic rat retina, the polyclonal CML antibody moderately stained the inner limiting membrane, the nerve fibre layer, and weakly stained the inner plexiform (Fig. 4c). The diabetic retina was strongly positive from the inner limiting membrane to the photoreceptor layer (Fig. 4 d). Of note, neovascular membranes which were partly present in the sections, were not labelled with any of AGE antibodies used.

Wheras AG-1 imidazolone antibody did not stain any part of the non-diabetic retina (Fig. $4 \mathrm{e}$ ), a moderate to strong immunolabelling was present in the inner part of the diabetic retina (Fig. 4f). In contrast to the diabetic rat retina, the staining was not limited to vascular structures.

The RAGE antibody moderately stained parts of the inner limiting membrane and the inner plexiform and nuclear layer (Fig. $4 \mathrm{~g}$ ), concordant with the distribution of GFAP (Fig. 4i). As GFAP in the normal retina is a marker of astrocytes, co-localization of RAGE and GFAP in normal retinas suggests expression in glial elements. A moderate signal in the diabetic retina which was not restricted to vascular structure (Fig. $4 \mathrm{~h}$ ) was observed. In the diabetic retina, the GFAP labelling extended from the inner limiting membrane to the outer nuclear layer, confirming previous results of a transdifferentiation Müller cells in diabetes [33].

\section{Discussion}

This study shows the distribution pattern of structurally defined AGE epitopes in diabetic retinopathy using well-characterized specific antibodies. We have 


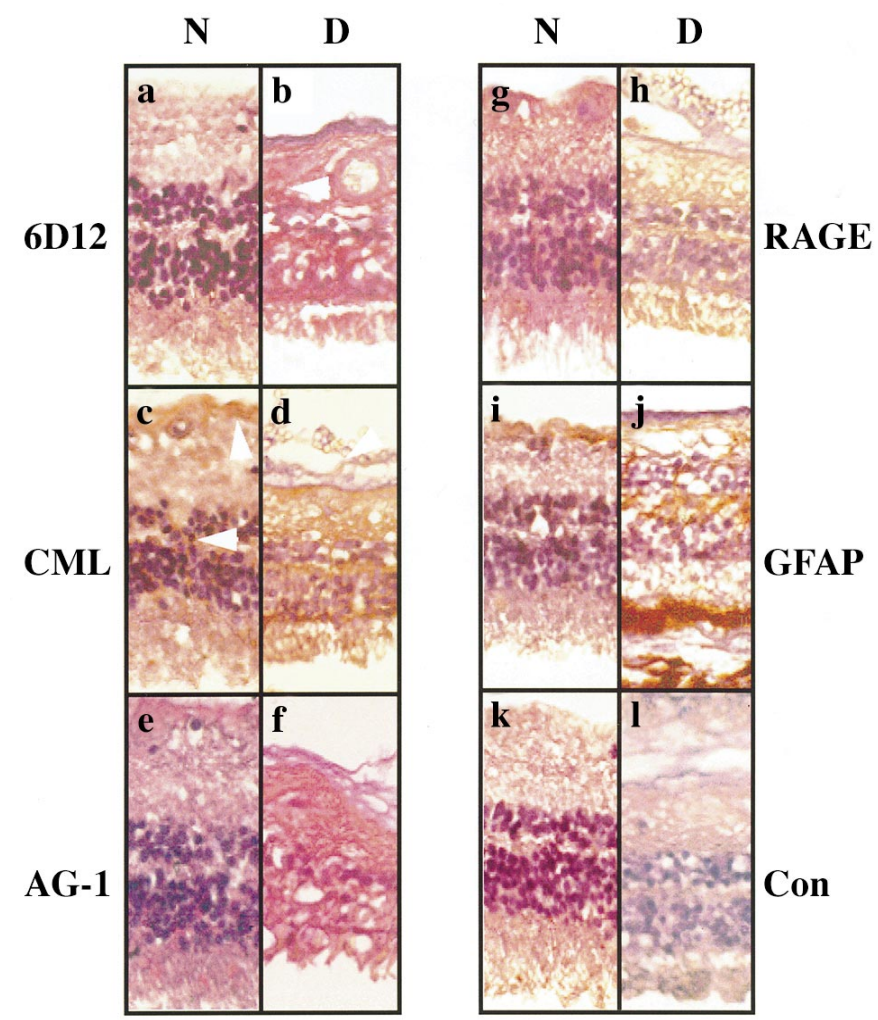

Fig.4.a-I Immunohistochemistry of a non-diabetic human retina $(\mathrm{N})$ and of a Type I diabetic patient (D) with proliferative retinopathy. a, b: normal (a) and diabetic (b) retina stained with the monoclonal 6D12 antibody. Note the difference and the strong immunolabelling of the inner plexiform layer (white arrow). c, d: normal (c) and diabetic (d) retina stained with the polyclonal CML antibody. c: Arrows mark positive labelling in the ganglion cell layer and in the inner nuclear layer. d: White arrow head marks a neovascular membrane that is negative for CML. e, f: normal (e) and diabetic (f) retina stained with the monoclonal AG-1 antibody, recognizing the imidazolone type AGE. g, h: Normal (g) and diabetic (h) retina stained with the polyclonal RAGE antibody. i, j: Normal (i) and diabetic (j) retina stained with the polyclonal GFAP antibody. Note the pronounced increase in labelling in the entire inner retina. k, l: Negative controls for normal (k), and diabetic retina (l). Original magnification $25 \times$

studied the spatial and, by indirect means, the temporal distribution of these AGE-epitopes in normal and diabetic retinas. Our results show that: (1) CML-immunoreactivity is present in the non-diabetic retina, with a pronounced increase in diabetes, (2) this immunoreactivity is present in neuroglial elements of diabetic and non-diabetic retinas, including photoreceptors with a pronounced increase in diabetes, (3) imidazolone-type AGE are exclusively located in the vasculature of the diabetic retina and differ in that respect from CML-type AGE, (4) imidazolone-type do not strictly co-localize with RAGE but CML-type protein modifications do co-localize with RAGE in the inner retina and (5) in advanced retinopathy, imidazolone-type and CML-type AGE are distributed throughout the whole retina. We chose the diabetic rat retina as representative of the early course of diabetic retinopathy. The diabetic rat lesions are characteristic of early background retinopathy in diabetic humans, in particular, pericyte loss and acellular capillaries. In contrast, the retina of a Type I diabetic patient with laser-treated proliferative diabetic retinopathy represents the end stage of the disease.

We describe the distribution and intensity of an imidazolone-type AGE in the diabetic retina. This antibody stained only vascular structures of the rat retina, consistent with the hypothesis that imidazolone-type AGE start to form in the inner vasculature in diabetes. Of note, during the advanced stage of retinopathy, the immunostaining spreads over the entire inner retina suggesting that both vascular and neuroglial parts of the retina are affected by AGE accumulation during the end stage. The staining pattern of the imidazolone-type AGE was more widespread than that of GFAP, a marker of Müller cells in diabetes, suggesting that additional cell population such as neuronal cells may accumulate AGE. Imidazolonetype AGE have been shown to be increased in erythrocytes of diabetic patients and were shown immunohistochemically in renal nodular glomerular lesions, in expanded mesangial matrix and in vessels of diabetic nephropathy [19].

In contrast to the imidazolone-type AGE, oxidation-dependent AGE like CML form as the consequence of both glycation and oxidation [21]. Our experiments characterizing the specificity of the CML antibodies showed that they did not cross-react with the non-oxidatively formed CEL and together with earlier results indicate that they specifically recognize CML. Results from the comparative dot blot analysis indicate that the 6D12 has a substantially lower affinity than the polyclonal CML antibody. The differences in the labelling pattern observed between these two antibodies could thus possibly be explained by the higher detection threshold of the 6D12, particularly evident in the non-diabetic rat retina, in which this antibody was negative. From these differences, it is concluded that the antibody affinity is of crucial importance in the evaluation of an AGE/CML antibody.

The polyclonal CML-antibody already labels retinal structures of the non-diabetic rat and human retina. Previous immunohistochemical studies using this antibody in non-diabetic humans reported on positive CML-staining in dermis, vessel walls, renal tubules and interstitium and intervertebral discs which was increased in diabetes and ageing [14]. In subjects less than 10 years of age CML-staining was rarely observed. We observed CML immunoreactivity, however, in retinas of 7.5-month-old non-diabetic rats. This unexpected finding indicates tissue-specific CML formation independent from hyperglycaemia and nonenzymatic glycation. Lipid peroxidation has been identified as an alternative source of CML formation [35] and is likely to occur in the retina since this tissue 
is rich in polyunsaturated fatty acids as a source of peroxidation in the presence of oxygen [36]. This could also explain the wide distribution of CML immunoreactivity in the non-diabetic retina, including the photoreceptor layer. CML immunoreactivity increased both in vascular and in non-vascular structures of diabetic rat and human retinas and was observed in the intracellular and extracellular compartment, indicative of augmented oxidative stress of the entire retina. Indeed, it has been reported that Müller cells become transdifferentiated to a GFAP- expressing cell type and heme oxygenase I, a heat-shock protein expressed in response to oxidative stress, is upregulated in the diabetic retina [37]. The up regulation of vascular endothelial growth factor and the low affinity binding site of nerve growth factor, p $75^{\mathrm{NGFR}}$, can further be related to the increased oxidative stress in diabetic retinas [38].

Others have reported on the localization of CML in retinas of non-diabetic and diabetic rats and humans. Results from one study [39] showed a much stronger immunoreactivity in the inner retina of formalin-fixed samples from non-diabetic SpragueDawley rats, compared with our results. Differences in rat strains, fixation techniques, antibody productions and staining procedures could account for the qualitative and quantitative differences observed between these data and ours. Using the 6D12 antibody, a staining pattern different from that reported here was shown in Type II diabetic patients with moderate degrees of retinopathy [40]. In that report, CML was restricted to the thickened vascular wall of vessels in the nerve fibre layer and to a strand-shaped staining pattern in the inner plexiform layer around large vessels. Shorter diabetes duration, lesser degrees of retinopathy and possibly also differences in the method could account for the less intense staining.

The predominant intracellular location of AGE occuring primarily in vascular structures agrees with the biochemical finding of an early and rapid increase of AGEs, possibly by highly reactive carbonyl compounds of glycolytic intermediates. By subtractive analysis (Table 2) of the oxidation-derived AGE compared with imidazolone-type AGE, it is evident that the vasculature is the primary location of this type of AGE. Inhibition of these AGE determined by autofluorescence with aminoguanidine reduces formation of acellular capillaries by $80 \%$ in diabetic rats, suggesting a pathogenetic role in diabetic retinopathy [15]. Aminoguanidine inhibits AGE formation by reacting with highly reactive dicarbonyl compounds such as 3 -deoxyglucosone with $\alpha, \beta$-diketones localized to proteins [41] and it reacts with methylglyoxal [42]. Glycoxidation product formation does not involve 3-deoxyglucosone intermediates. It is therefore unlikely that aminoguanidine would decrease CML formation. In line with this hypothesis, we have observed that in the rat model used in this study, am- inoguanidine does not decrease retinal CML immunoreactivity in diabetic rats and confirmed this results by immunoblotting (H.-P. Hammes, unpublished).

According to our findings, RAGE is upregulated in diabetic rats early during the course of diabetes and is primarily located in the inner retina and co-localizes with CML rather than with imidazolone-type AGE. Our data further suggest that one cell type, on which the RAGE is expressed is Müller cells. This receptor has also been found on vascular cells (endothelial cells and most likely pericytes) and its expression in up regulated in diabetes. According to the findings in the human retina, it appears that this upregulation is permanent and possibly increasing. Others have investigated the possible co-localization of CML with RAGE in diabetic rats and have found that RAGE is distributed over the whole retina including the photoreceptor layer, suggesting a complete co-localization with CML but no regulation in the presence of diabetes [39]. Again, the discrepancies between their study and ours probably results from technical differences, rat strains, antibody affinity and targets. Preliminary data indicate that CML-modified proteins, as recognized by the antibodies used in our study, are ligands for RAGE activating intracellular signalling pathways and altering gene expression [43].

Direct evidence for increased oxidative stress in this diabetic rat model has been provided in that heme oxygenase I (HSP 32) is up regulated in diabetic rats in a pattern reminiscent of GFAP staining which is indicative of Müller cells (see above). In contrast to the in-vitro situation, this increase cannot be abolished in diabetic rats with antioxdative treatment, which also does not ameliorate diabetic retinopathy [44].

In summary, we show that CML-like immunoreactivity is at a certain level a regular constituent of the normal retina, but increases in diabetes. Furthermore, we show the early presence of imidazolonelike activity restricted to the microvasculature which is primarily affected in early diabetes and an extension of this immunoreactivity during later stages. We suggest that the rat retina is a suitable target tissue for the in-vivo evaluation of drugs for the prevention of early diabetic retinopathy.

Acknowledgements. This work was supported in part by grants HA 1755/1-2 and Schl 239/6-2 from the Deutsche Forschungsgemeinschaft (German Research Society). The 6D12 antibody was kindly provided by Dr. S. Horiuchi and the RAGE antibody was a gift from Dr. J. Clausen (Copenhagen, Denmark).

\section{References}

1. Brownlee M (1994) Glycation and diabetic complications. Diabetes 43: 836-841

2. Bucala R, Cerami A (1992) Advanced glycation: chemistry, biology and implications in diabetes in aging. Adv Pharmacol 23: 1-34 
3. Hammes HP, Weiss A, Hess S et al. (1996) Modification of vitronectin by advanced glycation alters functional properties in-vitro and the diabetic retina. Lab Invest 75 : 325-338

4. Brownlee M, Cerami A, Vlassara H (1988) Advanced glycation end products in tissue and the biochemical basis of complications. N Engl J Med 318: 1315-1320

5. Eble AS, Thorpe SR, Baynes JW (1983) Nonenzymatic glucosylation and glucose-dependent cross-linking of proteins. J Biol Chem 258: 9506-9512

6. Fu MX, Knecht KJ, Thorpe SR, Baynes JW (1992) The role of oxygen in cross-linking and chemical modifications of collagen by glucose. Diabetes 41: 42-48

7. Sell DR, Lapolla A, Odetti P, Fogarty J, Monnier VM (1992) Pentosidine formation in skin correlates with severity of complication in individuals with long-standing IDDM. Diabetes 41: 1286-1292

8. Sell DR, Carlson EC, Monnier VM (1993) Differential effects of type 2 (noninsulin-dependent) diabetes mellitus on pentosidine formation in skin and glomerular basement membrane. Diabetes 40: 190-196

9. Schleicher E, Nerlich A (1996) The role of hyperglycaemia in the development of diabetic complications. Horm Metab Res 28: 367-373

10. Vlassara H, Brownlee M, Cerami A (1985) High-affinityreceptor-mediated uptake and degradation of glucosemodified proteins: a potential mechanism for the removal of senescent macromolecules. Procl Natl Acad Sci USA 82: $5588-5592$

11. Higashi T, Sano H, Saishoji T et al. (1997) The receptor for advanced glycation end products mediates the chemotaxis of rabbit smooth muscle cells. Diabetes 46: 463-472

12. Araki N, Ueno N, Chakraharti B, Morino Y (1992) Immunochemical evidence for the presence of advanced glycation end products in human lens proteins and its positive correlation with aging. J Biol Chem 267: 10211-10214

13. Monnier VM, Kohn RR, Cerami A (1984) Accelerated age-related browning of human collagen in diabetes mellitus. Proc Natl Acad Sci USA 81: 583-587

14. Schleicher ED, Wagner E, Nerlich AG (1997) Increased accumulation of the glycoxidation product $\mathrm{N}^{\varepsilon}$-(carboxymethyl)lysine in human tissues in diabetes and aging. $\mathrm{J}$ Clin Invest 99: 457-468

15. Hammes HP, Martin S, Federlin K, Geisen K, Brownlee M (1991) Aminoguanidine treatment inhibits the development of experimental diabetic nephropathy. Proc Natl Acad Sci USA 88: 11555-11558

16. Ledl F, Schleicher E (1990) New aspects of the Maillard reaction in foods and in the human body. Angew Chem Int Ed Engl 6: 565-594

17. Wells-Knecht KJ, Brinkmann E, Wells-Knecht MC et al. (1996) New biomarkers of Maillard reaction damage to proteins. Nephrol Dial Transplant 11 [Suppl 5] 41-47

18. Horie K, Miyata T, Maeda K et al. (1997) Immunohistochemical colocalization of glycoxidation products and lipid peroxidation products in diabetic renal glomerular lesions. J Clin Invest 100: 2995-3004

19. Niwa T, Katsuzaki T, Miyazaki S et al. (1997) Immunohistochemical detection of imidazolone, a novel advanced glycation end product, in kidneys and aortas of diabetic patients. J Clin Invest 99: 1272-1280

20. Niwa T, Katsuzaki T, Ishizaki Y et al. (1997) Imidazolone, a novel advanced glycation end product, is present at high levels in kidneys of rats with streptozotocin-induced diabetes. FEBS Lett 407: 297-302

21. Ahmed MU, Thorpe SR, Baynes JW (1986) Identification of $\mathrm{N}^{\varepsilon}$-(carboxymethyl)lysine as a degradation product of fructose lysine in glycated protein. J Biol Chem 261: 4889-4894

22. Smith PR, Thornally PJ (1992) Mechanism of the degradation of nonenzymatically glycated proteins under physiological conditions. Studies with a model fructosamine $\mathrm{N}^{\varepsilon}$ (1-deoxy-D-fructose-1-yl)hippuryl-lysine. Eur J Biochem 210: 729-739

23. Reddy S, Bichler J, Wells-Knecht KJ, Thorpe SR, Baynes JW (1995) $\mathrm{N}^{\varepsilon}$-(carboxymethyl)lysine is a dominant advanced glycation end product (AGE) in tissue proteins. Biochemistry 34: 10872-10878

24. Baynes JW (1991) Pespectives in diabetes, role of oxidative stress in development of complications in diabetes. Diabetes 40: 405-412

25. Wolff SP, Dean RT (1987) Glucose autooxidation and protein modification. The potential role of iautooxidative glycosylationì in diabetes. Biochem J 245: 243-250

26. Giardino I, Edelstein D, Brownlee M (1994) Nonenzymatic glycosylation in vitro and in bovine endothelial cells alters basic fibroblast growth factor activity. J Clin Invest 94: 110-117

27. Horiuchi S, Araki N, Morino Y (1991) Immunochemical approach to characterize advanced glycation end products of the Maillard reaction: Evidence for the presence of a common structure. J Biol Chem 266: 7329-7332

28. Ikeda K, Higashi T, Sano H et al. (1996) $\mathrm{N}^{\varepsilon}$-(carboxymethyl)lysine protein adduct is a major immunological epitope in proteins modified with advanced glycation end products of the Maillard reaction. Biochemistry 35: 8075-8083

29. Brett J, Schmidt AM, Yan SD et al. (1993) Survey of the distribution of an newly characterized receptor for advanced glycation end products in tissues. Am J Pathol 143: 1699-1712

30. Miyata T, Hori O, Zhang J et al. (1996) The receptor for advanced glycation end products (RAGE) mediates the interaction of AGE- $\beta 2$-microglobulin with human mononuclear phagocytes via an oxidant-sensitive pathway. Implications for the pathogenesis of dialysis-related amyloidosis. J Clin Invest 98: 1088-1094

31. Krook M, Ghosh D, Stromberg R, Carlquist M, Jornvall H (1993) Carboxyethyllysine in a protein: native carbonyl reductase/NADP( + )-dependent prostaglandin dehydrogenase. Proc Natl Acad Sci USA 90: 502-506

32. Neeper M, Schmidt AM, Brett J et al. (1992) Cloning and expression of a cell surface receptor for advanced glycosylation end products of proteins. J Biol Chem 267: 14998-15004

33. Mizutani M, Gerhardinger C, Lorenzi M (1998) Müller cell changes in human diabetic retinopathy. Diabetes 47: 445-449

34. Thornally PJ, Westwood M, Lo TW, McLellan AC (1995) Formation of methylglyoxal-modified proteins in vitro and in vivo and their involvement in AGE-related processes. Contrib Nephrol 112: 24-31

35. Fu MX, Requena JR, Jenkins AJ, Science TJ, Baynes JW, Thorpe SR (1996) The advanced glycation endproduct $\mathrm{N}^{\varepsilon}$ (carboxymethyl)lysine, is a product of both lipid peroxidation and glyoxidation reactions. J Biol Chem 271: 9982-9986

36. Ballabriga A (1994) Essential fatty acids and human tissue composition. An overview. Acta Paediatr Suppl 402: 63-68

37. Hammes HP, Bartmann A, Engel L, Wülfroth P (1997) Antioxidant treatment of experimental diabetic retinopathy in rats with nicanartine. Diabetologia 40: 629-634

38. Hammes HP, Federoff HJ, Brownlee M (1995) Nerve growth factor prevents both neuroretinal programmed cell death and capillary pathology in experimental diabetes. Mol Med 1: 527-534 
39. Soulis T, Thalass V, Youseff S et al. (1997) Advanced glycation end products and their receptors co-localise in rat organs susceptible to diabetic microvascular injury. Diabetologia 40: 619-628

40. Murata T, Nagai R, Ishibashi T, Inomata H, Ikeda K, Horiuchi S (1997) The relationship between accumulation of advanced glycation end products and expression of vascular endothelial growth factor in human diabetic retinas. Diabetologia 40: 764-769

41. Chen H-JC, Cerami A (1993) Mechanism of inhibition of advanced glycosylation by aminoguanidine in vitro. J Carbohydr Chem 12: 731-742

42. Araki A, Glomb MA, Takahashi M, Monnier VM (1998) Determination of dicarbonyl compounds as aminotriazines during the Maillard reaction and in vivo detection in aminoguanidine-treated rats. In: O'Brien J, Nursten HE, Crabbe MJC, Ames JM (eds) The Maillard reaction in foods and medicine. Royal Society of Chemistry, Cambridge, UK, 400 (Abstract)

43. Fu C, Pischetsrieder M, Hofmann M, Yan SF, Stern D, Schmidt AM (1998) Carboxymethyl-lysine advanced glycation endproduct modifications of proteins are ligands for RAGE that activate cell signalling pathways. Circulation 98: 1651 (Abstract)

44. Hammes HP, Weiss A, Führer D, Krämer HJ, Papavassilis C, Grimminger F (1996) Acceleration of the experimental diabetic retinopathy in the rat by omega-3 fatty acids. Diabetologia 39: 251-255 